\title{
Comparação da autopercepção da qualidade de vida relacionada a voz entre idosos participantes e não participantes de canto coral
}

\author{
Comparison of self-perceived voice-related quality of life among \\ elderly participants and non-participants in choir singing
}

\author{
Ana Caroline de Paula ${ }^{1}$ \\ Crismarie Casper Hackenberg ${ }^{2}$ \\ Sheila Beggiato ${ }^{3}$ \\ Valdomiro de Oliveira ${ }^{4}$ \\ Gislaine Cristina Vagetti ${ }^{5}$
}

\begin{abstract}
Resumo: A voz é um importante instrumento de comunicação que sofre alterações no processo de envelhecimento humano. Essas alterações podem impactar negativamente a qualidade de vida de pessoas idosas. O objetivo deste estudo foi comparar a autopercepção da qualidade de vida relacionada a voz em idosos praticantes e não praticantes de canto coral. Participaram da pesquisa 96 idosos com idade entre 60 e 90 anos, que responderam aos questionários sociodemográfico, socioeconômico e a versão brasileira do Protocolo de Qualidade de Vida em Voz (QVV). As médias de todos os grupos (canto coral e controle) apresentaram uma percepção positiva da qualidade de vida relacionado a voz geral, e nos aspectos físico e emocional. Nas comparações entre as médias de QVV dos grupos não foram encontradas diferenças estatisticamente significativas, indicando que os idosos participantes do estudo, mesmo sem participar de um grupo coral, podem apresentar uma autopercepção positiva quanto à sua voz e saúde vocal.
\end{abstract}

Palavras-chave: Idoso. Qualidade de vida. Voz. Canto. Linguística Aplicada.

Abstract: The voice is an important communication tool that undergoes changes in the human aging process. These changes can negatively impact the quality of life of elderly people. The aim of this study was to compare the self-perception of voice-related quality of life in elderly practitioners and non-practitioners of choir singing. The study included 96 elderly people aged between 60 and 90 years, who answered the sociodemographic, socioeconomic questionnaires and the Brazilian version of the Voice Quality of Life Protocol (V-RQOL). The averages of all groups (choral singing and control) showed a positive perception of quality of life related to the general voice, and in the physical and emotional aspects. In the comparisons between the mean QOL of the groups, no statistically significant differences were found, indicating that the elderly participants in the study, even without participating in a choir group, may present a positive self-perception regarding their voice and vocal health.

Keywords: Aged. Quality of life. Voice. Singing. Applied Linguistics.

\footnotetext{
1 Universidade Federal do Paraná, Setor de Educação, Curitiba, PR, Brasil. Endereço eletrônico: anacarolinedp@gmail.com.

2 Universidade Federal do Paraná, Setor de Educação, Curitiba, PR, Brasil. Endereço eletrônico: crismarie@ rioacapella.com.br.

${ }^{3}$ Universidade Estadual do Paraná, Campus Curitiba II, Centro de Área de Música e Musicoterapia, Curitiba, PR, Brasil. Endereço eletrônico: sheila.beggiato@unespar.edu.br.

4 Universidade Federal do Paraná, Setor de Educação, Curitiba, PR, Brasil. Endereço eletrônico: oliveira457@gmail.com.

${ }^{5}$ Universidade Estadual do Paraná, Campus Curitiba II, Centro de Área de Música e Musicoterapia, Curitiba, PR, Brasil. Endereço eletrônico: gislaine.vagetti@unespar.edu.br.
} 


\section{Introdução}

Nas últimas décadas, vem ocorrendo o aumento da expectativa de vida da população mundial, fato esse que gera novos debates e olhares para o envelhecimento e a própria velhice, pois, além de se viver mais, é necessário que se agregue qualidade aos anos vividos na maturidade (IBGE, 2018; VERAS; OLIVEIRA, 2018). A OMS (2005) tem proposto ações voltadas para um envelhecimento ativo e saudável, dirigindo um olhar global ao idoso, questionando ferramentas para manutenção da independência e autonomia na velhice como também ações de políticas públicas que contemplem a pessoa idosa, no que se refere, especialmente, à sua qualidade de vida e ao envelhecimento.

Esse processo natural da vida engloba uma complexidade de fatores fisiológicos, biológicos, sociais, cognitivos, emocionais que podem ser individuais e singulares, e ainda percebidos de forma objetiva e subjetiva. Nesse sentido, mesmo idosos saudáveis que não se encontram em um quadro de doenças e patologias, também sofrem perdas, declínios e transformações naturais da idade. Por esse motivo tratar o tema do envelhecimento é tão desafiador (ROLIM; FORTI, 2013; OMS, 2015; VERAS; OLIVEIRA, 2018).

É importante compreendermos a pessoa idosa a partir de seu próprio estilo de vida, das suas relações, expectativas, anseios, bem como por meio de suas percepções sobre diversos aspectos da vida. Dependendo da qualidade desses aspectos, positivos ou negativos, pode-se avaliar quais as necessidades desse público e definir quais ações assertivas podem ser ofertadas (GOMES; VAGETTI; OLIVEIRA, 2017).

Ações práticas e cotidianas dos idosos podem impactar na percepção de sua qualidade de vida (QV). A QV é um conceito amplo e pode ser entendido como a percepção que cada indivíduo tem de seus domínios físicos, psicológicos, sociais, relações com o ambiente, objetivos, expectativas, padrões e preocupações (WHOQOL-OLD, 1995).

Por ser tão abrangente, a QV pode ser estudada em especificidades, como nesta pesquisa, que se dedica a investigar a QV relacionada a voz (QVV). A QVV explora as percepções da própria voz, das transformações sofridas no decorrer da vida, bem como seu impacto nas relações sociais e em aspectos emocionais (PUTNOKI et al., 2010).

Estas alterações vocais geralmente acontecem devido a calcificação e ossificação das cartilagens da laringe, reduzindo a mobilidade e atrofiando os músculos intrínsecos, o que faz com o que o sistema fique menos eficiente e diminua a qualidade vocal. Estas mudanças são observadas aproximadamente entre os 60 e 70 anos de idade, sendo o estilo de vida um fator de impacto para a ocorrência dessas alterações (MARCHAND; BONAMIGO, 2015). 
Assim, os aspectos envolvidos no envelhecimento vocal podem modificar a sonoridade da voz falada e cantada. É possível observar diferenças entre vozes de jovens e idosos, pois o tom vocal, loudness (intensidade, amplitude) e qualidade sonora costumam ser afetados pelo envelhecimento. No caso de cantores é comum com o avanço da idade perceber-se soprosidade na voz, perda da amplitude, alterações nas vibrações, diminuição do controle respiratório e fadiga vocal (MARCHAND; BONAMIGO, 2015; MEIRELLES; BAK; CRUZ, 2012).

A presbifonia é um processo de envelhecimento vocal e pode também estar associada a casos depressivos, de ansiedade, isolamento social e diminuição da QV. Meirelles, Bak e Cruz (2012) ainda explicam que as mudanças vocais podem ocorrer por fatores hormonais. Em homens a frequência vocal pode aumentar aproximadamente $35 \mathrm{~Hz}$ por volta dos 65 anos, e em mulheres após a menopausa a frequência pode reduzir até $50 \mathrm{~Hz}$.

A audição e a fala são duas funções fortemente associadas a comunicação e ambas podem sofrer grandes alterações no processo de envelhecimento (KOST; SATALOFF, 2018). Para Aquino et al. (2016), tanto as desvantagens auditivas quanto as vocais poderão impactar negativamente na QV dos idosos, sendo importante mencionar que a autopercepção das alterações da fala pode passar despercebida aos idosos pela falta de demanda vocal intensa.

Observamos que idosos cantores apresentam vozes mais jovens quando comparados a idosos não cantores, e presume-se que isso se dá ao fato da realização de exercícios vocais regulares. Assim, cantar poderia prevenir ou atenuar mudanças associadas ao processo de envelhecimento vocal, considerado comum na velhice, mesmo não percebido e subnotificado. (KOST; SATALOFF, 2018).

Realmente a prática do canto pode desenvolver o controle respiratório, equilíbrio da ressonância, aumentar a tessitura vocal e promover cuidados com a voz. Cantar em um grupo pode gerar outros benefícios sociais e emocionais (AQUINO et al., 2016; KOST; SATALOFF, 2018). O canto coral é uma atividade que atua como profilaxia, ou seja, previne a perda de massa e tônus muscular da musculatura laríngea, gerando melhor condicionamento físico da voz. Além do bem-estar social e cultural, cantar também contribui para fortalecer os sistemas respiratório, auditivo e fonatório (MEIRELLES; BAK; CRUZ, 2012).

As atividades musicais têm um grande potencial para o desenvolvimento de habilidades e manutenção de aspectos cognitivos, sociais e emocionais. A música pode ser ainda uma ferramenta para as pessoas mais velhas se conectarem com seus sentimentos, identidade, empoderamento e desenvolvimento pessoal (CREECH et al., 2013).

A produção acadêmica sobre o canto coral para idosos é ainda muito recente, apresentando um vasto campo a ser investigado. Entre os anos de 1987 e 2017, apenas 8 
trabalhos do Banco de Teses e Dissertações da CAPES trataram dos dois temas canto coral e idosos, concomitantemente. Estes trabalhos, segundo a revisão de Bornholdt (2019), investigaram a temática por meio de três principais aspectos: psíquicos, físicos e sociais, oferecendo uma reflexão sobre o canto coral com idosos e favorecendo o tema no universo científico.

Diante do cenário exposto, esta pesquisa teve a seguinte questão norteadora: "participar de um grupo de canto coral pode beneficiar a autopercepção da QVV de pessoas idosas?”. Assim, o objetivo deste trabalho foi comparar a autopercepção de QVV entre idosos praticantes e não praticantes de canto coral.

\section{Método}

Este estudo seguiu as exigências da Resolução no 466/2012 do Conselho Nacional de Saúde (CNS), sendo aprovado pelo Comitê de Ética em Pesquisa (CEP) da Universidade Estadual do Paraná (UNESPAR) sob o parecer nº 3.077.043.

Trata-se de um estudo quantitativo, descritivo, comparativo e com delineamento transversal, explorando os dados de uma dissertação de mestrado em Educação. Foi realizado o cálculo amostral a priori, o número total da amostra foi estimado em 78 participantes divididos em 3 grupos de 26 participantes. Adotou-se o número mínimo de 30 participantes por grupo devido as possíveis desistências ou erros ao preencher os questionários, invalidando os dados. Foram respondidos 112 questionários, e destes, foram aceitos 96 por atenderem aos critérios de inclusão deste estudo.

Participaram 96 idosos residentes na cidade de Curitiba (Paraná), distribuídos em 3 grupos de atividades: a) grupo canto coral (GCC); b) grupo controle I - atividade física (GCI $\mathrm{AF})$; c) grupo controle II - não praticantes de canto coral e atividade física (GCII - NP).

Os critérios de inclusão adotados foram: homens e mulheres com idade igual ou superior a 60 anos, que aceitassem participar voluntariamente da pesquisa, assinassem o Termo de Consentimento Livre e Esclarecido (TCLE), participassem de um dos grupos supracitados. E os critérios de exclusão: homens e mulheres com idade inferior a 60 anos, que não assinassem o TCLE, não participassem dos grupos ou ainda participassem concomitantemente das atividades de canto coral e atividade física regular.

Sobre os grupos: a) GCC: idosos (homens e mulheres) que participassem de grupos corais amadores há no mínimo um ano, com ensaios regulares semanais de no mínimo 60 minutos, e não praticassem atividade física regular; b) GCI (AF): idosos (homens e mulheres) que participassem de atividades físicas estruturadas e coletivas, com acompanhamento 
profissional e não participassem de canto coral; c) GCII (NP): idosos (homens e mulheres) que participassem de grupos de convivência e não participassem dos grupos de canto coral e/ou atividade física. A fim de alcançar resultados mais fidedignos, foram escolhidos dois grupos controle, considerando a baixa produção de estudos que envolvam idosos e o canto coral.

A atividade física engloba todo e qualquer movimento realizado pelo corpo que requer gasto de energia, mesmo as atividades do dia a dia como tarefas domésticas, lazer, trabalho, e este termo muitas vezes é confundido com o exercício, que é planejado, estruturado e repetitivo, a fim de manter ou melhorar a aptidão física (WHO, 2018). Entende-se que todos os idosos do estudo realizam atividade física, inclusive os coralistas e participantes dos grupos de convivência, aqui se enquadram no grupo "atividade física” aqueles que realizam exercício físico regularmente sob orientação e supervisão de profissionais qualificados.

A coleta de dados aconteceu de março a novembro de 2019 e foram utilizados para esta finalidade questionários autoaplicados. Para a caracterização sociodemográfica e socioeconômica foram utilizados: questionário sociodemográfico adaptado do Brazil Old Age Schedule (BOAS) desenvolvido por Veras e Dutra (2008) e questionário de classificação econômica (ABEP, 2018). Para investigar a autopercepção da qualidade de vida em voz foi utilizado o Protocolo de Qualidade de Vida em Voz (QVV) que gera a pontuação total, e os escores físico e emocional, validado no Brasil por Behlau, Hogikyan e Gasparini (2007).

O Protocolo de Qualidade de Vida em Voz é composto por dez itens, sendo seis referentes ao domínio físico e quatro de domínio socioemocional. As questões são respondidas em uma escala de cinco pontos, na qual 1 se refere à "nunca acontece e não é um problema", 2 à "acontece pouco e raramente é um problema", 3 à "acontece às vezes e é um problema moderado", 4 à "acontece muito e quase sempre é um problema", e 5 à "acontece sempre e realmente é um problema ruim". O cálculo utiliza um algoritmo padrão que pode variar de $0 \mathrm{a}$ 100, sendo que 100 indica melhor percepção da qualidade de vida possível (BEHLAU; HOGIKYAN; GASPARINI, 2007).

Para a análise de dados foi utilizado o software estatístico Stata MP 14.1, realizando comparações múltiplas por meio do post hoc de Bonferroni e foi adotado $\mathrm{p}<0,05$ para a significância estatística. A descrição dos participantes foi realizada mediante medidas de tendência central e dispersão para as variáveis quantitativas e distribuição de frequência absoluta para as variáveis qualitativas. As análises comparativas foram realizadas por meio de ANOVA para 1 fator, no caso de não cumprimento do pressuposto de homogeneidade de variâncias foi utilizado o Welch test. 


\section{Resultados}

\section{Caracterização dos participantes}

A idade média dos participantes foi de 70,10 \pm 7,61 anos, sendo o mínimo 60 anos e máximo 90 anos, com maior concentração $(56,25 \%)$ na faixa etária de 62 a 78 anos. Todos os participantes eram brasileiros (100\%), maioria do gênero feminino $(84,38 \%)$, de cor/raça branca $(89,58 \%)$, naturais do Paraná $(51,04 \%)$, de estado civil casado $(48,96 \%)$, escolaridade máxima ensino médio completo (20,83\%) e superior completo (20,83\%), com arranjo familiar acompanhados $(73,96 \%)$, com predominância de classe econômica C1 (28,12\%), aposentados $(74,04 \%)$, sendo a aposentadoria a principal remuneração $(73,96 \%)$. As informações sociodemográficas e socioeconômicas são apresentadas na Tabela 1.

Tabela 1 - Características sociodemográficas e socioeconômicas dos participantes (N=96), Curitiba, PR, Brasil

\begin{tabular}{|c|c|c|}
\hline & $\mathbf{N}$ & $\%$ \\
\hline \multicolumn{3}{|l|}{ Gênero } \\
\hline Masculino & 15 & 15,62 \\
\hline Feminino & 81 & 84,38 \\
\hline \multicolumn{3}{|l|}{ Cor/raça } \\
\hline Branca & 86 & 89,58 \\
\hline Preta & 0 & 0,00 \\
\hline Amarela & 4 & 4,17 \\
\hline Parda & 6 & 6,25 \\
\hline \multicolumn{3}{|l|}{ Naturalidade } \\
\hline Bahia & 1 & 1,04 \\
\hline Paraná & 49 & 51,04 \\
\hline Pernambuco & 1 & 1,04 \\
\hline Rio Grande do Sul & 6 & 6,25 \\
\hline Rio de Janeiro & 3 & 3,12 \\
\hline Santa Catarina & 14 & 14,58 \\
\hline São Paulo & 8 & 8,33 \\
\hline Não informado & 14 & 14,58 \\
\hline \multicolumn{3}{|l|}{ Estado Civil } \\
\hline Casado(a) & 47 & 48,96 \\
\hline Viúvo(a) & 27 & 28,12 \\
\hline Divorciado(a)/Separado(a) & 10 & 10,42 \\
\hline Nunca casou & 12 & 12,50 \\
\hline \multicolumn{3}{|l|}{ Escolaridade } \\
\hline Analfabeto & 1 & 1,04 \\
\hline Primário Incompleto & 6 & 6,25 \\
\hline
\end{tabular}


Primário Completo/Ensino Fundamental

Ensino Fundamental Completo/Ensino Médio Incompleto

Ensino Médio Completo/ Superior Incompleto

Superior Completo

Curso Técnico Incompleto

Curso Técnico Completo

Pós-Graduação

\section{Arranjo Familiar}

Sozinho(a)

Acompanhado(a)

\section{Classificação Econômica}

A

B1

B2

C1

$\mathrm{C} 2$

D-E

\section{Principal Remuneração}

Aposentadoria

Emprego

Outro: pensão, auxílio, bolsa

$\begin{array}{ll}14 & 14,58 \\ 13 & 13,54 \\ 20 & 20,83 \\ 20 & 20,83 \\ 1 & 1,04 \\ 4 & 4,17 \\ 17 & 17,71 \\ & \\ 25 & 26,04 \\ 71 & 73,96\end{array}$

$17 \quad 17,71$

$10 \quad 10,42$

$26 \quad 27,08$

$27 \quad 28,12$

99,38

$\begin{array}{ll}7 & 7,29\end{array}$

$71 \quad 73,96$

$5 \quad 5,21$

$20 \quad 20,83$

Fonte: elaborada pelos autores.

Qualidade de vida em voz

Quanto a autopercepção da QVV, as comparações dos escores dos grupos são apresentadas na Tabela 2. Nenhuma diferença significativa foi verificada entre os grupos.

Tabela 2 - Comparação da autopercepção de qualidade de vida em voz entre os idosos dos grupos de canto coral, controle I (Af), controle II (Np) (N=96), Curitiba, PR, Brasil

\begin{tabular}{llllll}
\hline & $\begin{array}{l}\text { Canto Coral } \\
(\mathrm{n}=32)\end{array}$ & Controle I $(\mathrm{n}=32)$ & $\begin{array}{l}\text { Controle } \\
(\mathrm{n}=32)\end{array}$ & II & \\
\hline & Média \pm DP & Média \pm DP & Média \pm DP & F & P \\
\hline Qualidade de Vida Geral & $91,02 \pm 11,49$ & $92,03 \pm 11,10$ & $91,72 \pm 16,31$ & 0,05 & 0,96 \\
Sócio-Emocional & $92,38 \pm 12,37$ & $94,92 \pm 11,05$ & $96,09 \pm 13,91$ & 0,74 & 0,48 \\
Funcionamento Físico & $90,10 \pm 1,19$ & $90,10 \pm 11,73$ & $88,80 \pm 19,21$ & $0,06^{*}$ & 0,94 \\
\hline
\end{tabular}

DP: Desvio-padrão. * Welch test.

Fonte: elaborada pelos autores. 


\section{Discussão}

Este estudo buscou comparar a autopercepção da QVV entre idosos praticantes e não praticantes de canto coral. Quanto ao perfil dos participantes a média de idade foi de 70,10 \pm 7,61 anos, tendo a maior concentração na faixa etária entre 62 e 78 anos. Quanto à cor/raça, idade e estado civil, a predominância foi de pessoas brancas, casadas e com idade média próxima aos 70 anos, assim como nos estudos de Vagetti et al. (2015); Krawutschke (2017); Arruda (2018); Flores-Gomes (2019), realizados na cidade de Curitiba/Paraná. Houve uma participação expressiva de mulheres $(84,38 \%)$, o que pode ocorrer pela maior longevidade feminina e pela maior participação deste gênero em atividades sociais (PINTO; NERI, 2017).

Quanto a escolaridade a maioria possuía ensino médio completo $(20,83 \%)$ e ensino superior completo $(20,83 \%)$, e a principal fonte de renda observada foi a aposentadoria, assim como nos estudos supracitados. O perfil dos participantes diferencia-se, especificamente, dos estudos de Arruda (2018) e Flores-Gomes (2019) na questão da classificação econômica, em que a maioria estava inserida na classe B.

Quanto a comparação da autopercepção da QVV, não foram encontradas diferenças estatisticamente significativas entre os grupos de canto coral e os dois grupos controle. O que de acordo com Godoy (2016) esses achados podem ocorrer pois os participantes encontram-se em uma relação positiva e saudável com sua saúde vocal.

As médias dos grupos indicaram que os participantes apresentaram uma autopercepção positiva de voz e os resultados enquadram-se dentro de um perfil de voz saudável, de acordo com o protocolo utilizado. O protocolo QVV identifica, por meio da autoavaliação, a presença da disfonia, sendo que em caso de disfonia os escores totais são iguais ou inferiores a 71,6, no escore socioemocional 79,5 e 74,9 no escore físico (KOST, SATALOFF, 2018; BEHLAU et al., 2009), no presente estudo, todas as médias dos grupos foram superiores a estes indicadores.

Behlau et al. (2009) ressaltam que o protocolo QVV não é repetitivo, contempla os escores físico e emocional, tem uma base de fácil compreensão, possui uma questão destinada a profissão (que muitas vezes impacta a QVV, como no caso de professores). $\mathrm{O}$ uso deste protocolo foi escolhido devido a sua fácil compreensão e aplicação, tendo em vista que foram autoaplicados pelos idosos participantes.

Estudos de Godoy (2016), Penteado e Penteado (2010), Schneider et al. (2010), Gampel, Karsch, Ferreira (2010), também utilizaram este protocolo em pesquisas com pessoas idosas, e não encontraram diferenças estatisticamente significativas. Ressalta-se, contudo, que no protocolo não há nenhuma questão específica sobre aspectos da performance do canto em relação a voz. 
Sobre a autopercepção da QVV, Godoy (2016) comparou um grupo de Terapia Vocal para Idosos com um grupo de não participantes e não encontrou diferenças estatísticas significativas. Observou-se que o grupo que participou da terapia vocal apresentou aumento nas médias dos domínios físicos e socioemocionais do protocolo. No estudo de Godoy (2016), o canto não foi ferramenta de intervenção, mas houve uso de escalas musicais e vocalizes pequenas frases musicais.

No estudo de Penteado e Penteado (2010) participaram apenas idosos cantores de um grupo coral e explorou-se a imagem vocal e cuidados com a saúde da voz. Foi observado que 9 em 10 idosos coralistas tiveram uma percepção positiva em relação a sua voz. Por meio da avaliação fonoaudiológica foi possível constatar alterações discretas em parâmetros vocais dos participantes o que não influenciou na percepção positiva dos coralistas. Penteado e Penteado (2010) ressaltam que a presença da autopercepção vocal positiva mesmo com a avaliação fonoaudiológica apresentando alterações vocais em grau leve pode ocorrer em idosos coralistas e não coralistas, porém em coralistas o impacto tende a ser menor.

O estudo de Schneider et al. (2010) com idosos alemães relatou que é possível que as pessoas idosas não percebam alterações vocais no processo de envelhecimento tanto quanto percebem outras alterações físicas. Com isso é mais difícil a autopercepção sem um exame vocal específico que identifique essas alterações. Os participantes também tiveram uma autopercepção positiva em relação a voz.

Gampel, Karsch e Ferreira (2010) compararam a autopercepção vocal de idosos professores e não professores, pois os professores costumam apresentar ao longo da carreira problemas relacionados ao domínio físico da voz. Porém as comparações da autopercepção de QVV não obtiveram diferenças estatísticas, e os professores declararam maior percepção de alterações físicas da voz. Os não professores afirmaram que as alterações têm impacto tanto em aspectos físicos quanto socioemocionais.

Os estudos supracitados não compararam a QVV entre idosos praticantes de canto coral e não praticantes, mas trazem achados importantes para a presente discussão. Os autores avaliam que o uso do protocolo QVV é adequado ao público idoso, pois permite uma rápida investigação sobre aspectos físicos e emocionais da saúde vocal. Os mesmos autores concordam que uma boa percepção da saúde vocal nem sempre reflete os aspectos físicos em si, pois muitas vezes, os idosos criam estratégias adaptativas do seu uso de voz no processo de envelhecimento vocal não percebendo o seu impacto no cotidiano.

No presente estudo, como apresentado anteriormente, considerou-se o uso deste protocolo como adequado ao grupo participante, devido a sua aplicabilidade e compreensão por 
parte dos idosos. Ressaltamos que um instrumento de QVV, que considere aspectos da voz cantada, poderia observar diferentes percepções comparando-se praticantes e não praticantes de canto coral.

A música alcança todas as pessoas no mundo de forma subjetiva. Apesar de uma pessoa não participar da atividade de canto coral, sua musicalidade poderia estar presente de diversas maneiras em sua vida cotidiana. Podemos cantar em casa acompanhando as canções tocadas nas rádios e outros canais midiáticos ou ainda cantar em eventos religiosos e sociais, nos emocionarmos profundamente com música e não sermos coralistas.

Neste estudo com praticantes e não praticantes do canto coral observou-se que há um grande desvio padrão nas médias, o que indica que os grupos não foram homogêneos quanto sua autopercepção da QVV. O grupo controle I apresentou as maiores médias em todos os aspectos investigados, já o grupo controle II apresentou todas as menores médias, e estas mais próximas aos sinais de disfonia.

A voz é um dos aspectos que impacta a percepção geral da QV e da saúde, como apresentado por Almeida (2013) e Yinger (2014), cantar em grupo coral irá envolver inúmeros aspectos físicos, psicológicos, emocionais e sociais. Nesse sentido, Kost e Sataloff (2018) afirmam que os distúrbios vocais prejudicam a capacidade de comunicação com os familiares, amigos e as relações sociais em si.

Lortie et al. (2016) e Kost e Sataloff (2018) reforçam que a prática do canto, seja ele individual ou coletivo, pode prevenir, diminuir ou retardar o envelhecimento vocal de maneira não invasiva, impactando positivamente a comunicação das pessoas idosas. Os autores ressaltam que a prática do canto coletivo favorece os aspectos socioemocionais.

Segundo Almeida (2013), nos grupos de canto coral, o professor, regente e preparador vocal têm um papel importante na observação dos sinais apresentados pelos idosos. O ensaio é um espaço pedagógico e de observação acústica para se buscar estratégias que fortaleçam e potencializem as habilidades vocais dos praticantes de canto coral. Essas estratégias adaptativas que irão permitir um ambiente de aprendizado e desenvolvimento musical são reforçadas por Yinger (2014) como uma oportunidade de atenção e observação de possíveis perdas e danos nos sentidos dos idosos.

Aquino et al. (2016), Lortie et al. (2016) e Kost e Sataloff (2018) reforçam que além de avaliar a QVV também são necessárias ferramentas que possam auxiliar na promoção da QVV. $\mathrm{O}$ canto poderia ser uma destas ferramentas protetivas atenuando sinais da presbifonia e evitando o uso de medicamentos e procedimentos cirúrgicos para reverter possíveis danos no 
trato vocal. Para isto, mais estudos deveriam explorar a percepção subjetiva sobre a QVV, realizando testes fonoaudiológicos que verificassem os aspectos físicos da saúde vocal.

\section{Considerações finais}

A voz continua sendo uma importante ferramenta de comunicação e integração social. As alterações vocais sofridas no processo de envelhecimento podem prejudicar a autoimagem, as relações socioemocionais, até mesmo ser um fator que impacta na percepção negativa de saúde e QV. Este estudo buscou investigar esse efeito na população idosa comparando a autopercepção da qualidade de vida relacionada à voz entre idosos praticantes e não praticantes de canto coral.

Optou-se por utilizar dois grupos controle para maior fidedignidade aos dados, considerando que poucos estudos que investiguem a QVV de idosos foram encontrados. Uma limitação do estudo foi o fato de muitos idosos atualmente participarem concomitantemente de atividades de canto coral e atividade física estruturada, e com isso, vários questionários terem sido excluídos.

A literatura vem apresentando o canto coral como uma prática benéfica para a saúde física, emocional e social. A QVV não apresentou diferenças estatisticamente significativas entre os grupos de idosos praticantes e não praticantes de canto coral. As médias dos grupos enquadram-se em uma autopercepção positiva de QVV. Podemos considerar que a autopercepção vocal pode ser um bom indicativo para compreender e melhorar o atendimento de pessoas idosas.

Os estudos apresentados na discussão demonstraram que nas avaliações fonoaudiológicas e nas observações dos profissionais que atuam com grupos foram constatados fortes sinais de alterações da voz na fase do envelhecimento. Importante relevar que por mais que os participantes apresentem uma boa relação com sua saúde vocal esta percepção pode não condizer com a realidade da voz em si. Reforça-se a necessidade da continuidade de novos estudos sobre os aspectos da voz no decorrer do envelhecimento e a associação com promoção da saúde vocal.

\section{Referências}

ABEP. Associação Brasileira de Empresas de Pesquisa. Critério de classificação econômica do Brasil. São Paulo: Associação Brasileira de Empresas de Pesquisa, 2018.

ALMEIDA, M. C. P. de. O canto coral e a terceira idade - o ensaio como momento de grandes possibilidades. Revista da ABEM, Londrina, v. 21, n. 31, p. 119-133. 2013. 
Disponível em:

http://www.abemeducacaomusical.com.br/revistas/revistaabem/index.php/revistaabem/article/ view/77. Acesso em: 14 jul. 2021.

AQUINO, F. S. et al. Características da voz falada de idosas com prática de canto coral. CoDAS, São Paulo, v. 28, n. 4, p. 446-453, 2016. Disponível em: https://doi.org/10.1590/2317-1782/20162015109. Acesso em: 14 jul. 2021.

ARRUDA, M. L. A influência da Musicoterapia na percepção da qualidade de vida de pessoas idosas. 2018. 121 f. Dissertação (Mestrado em Educação) - Programa de PósGraduação em Educação, Universidade Federal do Paraná, Curitiba, 2018.

BEHLAU, M.; HOGIKYAN, N. D.; GASPARINI, G. Quality of Life and Voice: Study of a Brazilian Population Using the Voice-Related Quality of Life Measure. Folia Phoniatrica et Logopaedica, v. 59, p. 286-296, 2007. Disponível em: https://doi.org/10.1159/000108335. Acesso em: 14 jul. 2021.

BEHLAU, M. et al. Validação no Brasil de protocolos de auto-avaliação do impacto de uma disfonia. Pró-Fono Revista de Atualização Científica, Barueri, v. 21, n. 4, p. 326-332. 2009. Disponível em: http://dx.doi.org/10.1590/S0104-56872009000400011. Acesso em: 14 jul. 2021.

BORNHOLDT, J. H. Canto coral com idosos: o que falam os regentes e as rotinas de ensaio. 2019. 149 f. Dissertação (Mestrado em Música) - Programa de Pós-Graduação em Música, Universidade Federal do Paraná, Curitiba, 2019.

CREECH, A. et al. The power of music in the lives of older adults. Research Studies in Music Education, v. 35, n. 1, p. 83-98, 2013. Disponível em: https://doi.org/10.1177/1321103X13478862. Acesso em: 14 jul. 2021.

FLORES-GOMES, G. F. Efeitos de um programa de inclusão digital nas funções cognitivas e qualidade de vida de idosos. 2019. $181 \mathrm{f}$. Dissertação (Mestrado em Educação) - Programa de Pós-Graduação em Educação, Universidade Federal do Paraná, Curitiba, 2019.

GAMPEL, D.; KARSCH, U. M.; FERREIRA, L. P. Percepção de voz e qualidade de vida em idosos professores e não professores. Ciência e saúde coletiva, v. 15, n. 6, p. 2907-2916, 2010. Disponível em: https://doi.org/10.1590/S1413-81232010000600028. Acesso em: 14 jul. 2021.

GODOY, J. F. Efetividade do programa Terapia Vocal para Idosos nas formas convencional e intensiva. 2016. $111 \mathrm{f}$. Tese (Doutorado em Fonoaudiologia) - Programa de Pós-Graduação em Fonoaudiologia, Faculdade de Odontologia de Bauru, Universidade de São Paulo, Bauru, 2016.

GOMES, F. R. H.; VAGETTI, G. C.; OLIVEIRA, V. Envelhecimento humano: cognição, qualidade de vida e atividade física. Appris: Curitiba, 2017.

IBGE. Projeções da população do Brasil e Unidades da Federação por sexo e idade 2010-2060: revisão 2018. 2. ed. Rio de Janeiro: IBGE, 2018. 
KOST, K. M.; SATALOFF, R. T. Voice disorders in the elderly. Clinics in Geriatric Medicine, v. 34, n. 2, p. 191-203, 2018. Disponível em:

https://doi.org/10.1016/j.cger.2018.01.010. Acesso em: 14 jul. 2021.

KRAWUTSCHKE, A. Percepção de qualidade de vida em idosas que exercem e não exercem a prática corporal. 2017. 89 f. Dissertação (Mestrado em Educação) - Programa de Pós-Graduação em Educação, Universidade Federal do Paraná, Curitiba, 2017.

LORTIE, C. et al. The moderating effect of frequent singing on voice aging. Journal of Voice, v. 31, n. 1, p. 112-124, 2016. Disponível em: https://doi.org/10.1016/j.jvoice.2016.02.015. Acesso em: 14 jul. 2021.

MARCHAND, D. L. P.; BONAMIGO, A. W. Atuação fonoaudiológica na voz do idoso: revisão sistemática exploratória de literatura. Distúrbios da Comunicação, São Paulo, v. 27, n. 2, p. 309-317, 2015.

MEIRELLES, R. C.; BAK, R.; CRUZ, F, C. Presbifonia. Revista do Hospital Universitário Pedro Ernesto, Rio de Janeiro, v. 17, n. 2, p. 77-82, 2012.

OMS. Envelhecimento Ativo: uma Política de saúde. Brasília: Organização Pan-Americana da Saúde, 2005.

OMS. Resumo: relatório mundial de envelhecimento e saúde. Organização Mundial da Saúde. Tradução não informada. Suíça, 2015. Disponível em: https://sbgg.org.br/wpcontent/uploads/2015/10/OMS-ENVELHECIMENTO-2015-port.pdf. Acesso em: 14 jul. 2021.

PENTEADO, R. Z.; PENTEADO, L. A. P. B. Percepção da voz e saúde vocal em idosos coralistas. Revista CEFAC, São Paulo, v. 12, n. 2, 2010. Disponível em: https://doi.org/10.1590/S1516-18462009005000053. Acesso em: 14 jul. 2021.

PINTO, J. M.; NERI, A. L. Trajetórias da participação social da velhice: uma revisão sistemática da literatura. Revista Brasileira de Geriatria e Gerontologia, Rio de Janeiro, v. 20, n. 2, 2017. Disponível em: http://dx.doi.org/10.1590/1981-22562017020.160077. Acesso em: 14 jul. 2021.

PUTNOKI, D. S. et al. Qualidade de vida em voz: o impacto de uma disfonia de acordo com gênero, idade e uso vocal profissional. Rev. Soc. Bras. Fonoaudiol., São Paulo, v. 15, n. 4, p. 485-490, 2010.

ROLIM, F. S.; FORTI, V. A. M. Envelhecimento e atividade física: auxiliando na melhoria e manutenção da qualidade de vida. In: DIOGO, M. J. D.; NERI; A. L; CACHIONI, M.

(Orgs.). Saúde e qualidade de vida na velhice. 4. ed. Campinas: Editora Alínea, 2013. p. 5773.

SCHNEIDER, S. et al. Voice Function and Voice-Related Quality of Life in the Elderly. Gerontology, v. 57, p. 109-114, 2011. Disponível em: https://doi.org/10.1159/000314157. Acesso: 14 jul. 2021. 
VAGETTI, G. C. et al. The association between physical activity and quality of life domains among older women. Journal of Aging and Physical Activity, v. 23, n. 4, p. 524-533, 2015. Disponível em: https://doi.org/10.1123/japa.2013-0070. Acesso em: 14 jul. 2021.

VERAS, R.; DUTRA, S. Perfil do idoso brasileiro: questionário BOAS. Rio de Janeiro: UERJ, UnATI, 2008.

VERAS, R.; OLIVEIRA, M. Envelhecer no Brasil: a construção de um modelo de cuidado. Ciência \& Saúde Coletiva, Rio de Janeiro, v. 23, n. 6, p. 1929-1936, jun. 2018. Disponível em: https://doi.org/10.1590/1413-81232018236.04722018. Acesso em: 14 jul. 2021.

WHO. Global action plan on physical activity 2018-2030: more active people for a healthier world. Geneva, World Health Organization, 2018.

WHOQOL. The World Health Organization Quality of Life Assessment (WHOQOL): Position paper from the World Health Organization. Social Science \& Medicine, v. 41, n. 10, p. 1403-1410, 1995.

YINGER, O. S. Adapting choral singing experiences for older adults: the implications of sensory, perceptual, and cognitive changes. International Journal of Music Education, v. 32, n. 2, p. 203-212, 2014. Disponível em: https://doi.org/10.1177/0255761413508064. Acesso em: 14 jul. 2021. 


\section{Sobre os autores}

Ana Caroline de Paula (Orcid iD: http://orcid.org/0000-0002-2536-6848)

Mestra em Educação pela Universidade Federal do Paraná (UFPR); graduada em Licenciatura em Música pela Universidade Estadual do Paraná - Campus Curitiba I (UNESPAR-EMBAP).

Crismarie Casper Hackenberg (Orcid iD: http://orcid.org/0000-0003-3838-0769) Doutoranda em Educação pela Universidade Federal do Paraná (UFPR); mestra em Psicologia pela Universidade Católica de Petrópolis (UCP); pós-graduada em Neurociências Aplicadas a Aprendizagem pela Universidade Federal do Rio de Janeiro (UFRJ); graduada em Educação Artística pelo Conservatório Brasileiro de Música - Rio de Janeiro (CBM/RJ) e em Pedagogia pela Universidade Estácio de Sá (UNESA).

Sheila Beggiato (Orcid iD: http://orcid.org/0000-0002-5207-3362)

Doutoranda em Educação pela Universidade Federal do Paraná (UFPR); mestra em Educação pela Pontifícia Universidade Católica do Paraná (PUC-PR); graduada em Musicoterapia pela Universidade Estadual do Paraná (UNESPAR). É professora da Universidade Estadual do Paraná (UNESPAR), Campus Curitiba II.

Valdomiro de Oliveira (Orcid iD: https://orcid.org/0000-0002-8709-8471)

Doutor e mestre em Educação Física pela Universidade Estadual de Campinas (UNICAMP); graduado em Educação Física pela Universidade Estadual de Maringá (UEM). É professor do Programa de Pós-Graduação em Educação da Universidade Federal do Paraná.

Gislaine Cristina Vagetti (Orcid iD: http://orcid.org/0000-0003-0704-1297)

Doutora em Educação Física pela Universidade Feral do Paraná (UFPR); mestra em Ciências da Saúde pela Universidade Estadual de Maringá (UEM); graduada em Educação Física pela UEM. É professora do Programa de Pós-Graduação em Educação da Universidade Estadual do Paraná (UNESPAR), Campus Curitiba II.

Recebido em abril de 2021

Aprovado em maio de 2021. 